\title{
Understanding the hepatitis B core positive liver donor
}

\author{
Kah Wai Clarence $\underline{K w a n}^{1,2}$, MBBS, MRCP, Teegan Reina $\underline{\operatorname{Lim}}^{1}$, MBChB, MRCP, Rajneesh $\underline{K u m a r}^{1,3}$, MD, MRCP, \\ Thinesh Lee Krishnamoorthy, ${ }^{1,3}$, MBChB, MRCP
}

\begin{abstract}
The increasing number of patients on the Singapore national liver transplant waiting list and the lack of donor livers have necessitated a review of the limited use of marginal donor liver grafts. Some grafts are of good quality but are considered marginal due to positive donor antibody to hepatitis B virus core protein serology, and negative hepatitis $B$ surface antigen (HBsAg) and hepatitis B DNA. The fear is of viral reactivation during periods of intense immunosuppression. This is made possible by the ability of the hepatitis $B$ virion to reside in a dormant state within the hepatocyte nucleus despite HBsAg clearance, i.e. the occult hepatitis B infection (OBI). In truth, appropriate selection of recipients and effective post-transplantation immunoprophylaxis significantly reduce the risk of hepatitis $B$ viral reactivation. This article explains the confusion surrounding $\mathrm{OBI}$ and reviews current recommendations on how to manage such donor liver grafts.
\end{abstract}

Keywords: core positive donor, liver transplant, occult hepatitis $B$, viral reactivation

\section{INTRODUCTION}

End-stage liver disease carries high mortality and morbidity, with liver transplantation being the only form of definitive treatment. In Singapore, despite state laws for mandatory organ donation and efforts to improve national awareness, the availability of liver grafts remains low. Based on statistics released by the Ministry of Health, Singapore, the number of patients on the national liver transplant waiting list tripled over a five-year period between 2007 and 2012, with an average of seven waiting-list patient deaths each year. ${ }^{(1)}$

Graft availability is a common issue worldwide. In view of this, novel approaches to expand the graft supply have been experimented on and redefined over the years. One strategy involves the use of 'marginal' grafts from donors who have antibodies against hepatitis B virus core protein (anti-HBc) but test negative for hepatitis B surface antigen ( $\mathrm{HBsAg})$. These are commonly referred to as hepatitis B 'core positive donors'. This is of clinical relevance, especially in Asia-Pacific countries like Singapore, where hepatitis B virus (HBV) infection remains endemic and the local anti-HBc positive rate is $22.5 \%$. $^{(2)}$

This article aims to: (a) review the HBV life cycle and the role(s) of covalently closed circular DNA (cccDNA) to improve understanding of the differences between anti-HBc positivity and occult HBV infection (OBI); and (b) highlight key points to ensure the safe utilisation of liver grafts from core positive donors and minimise the risk of de novo HBV infection (DNHI).

\section{OVERVIEW OF HBV LIFE CYCLE}

\section{Structure of hepatitis B virion or 'Dane particles'}

Infectious virions (Fig. 1) comprise an outer envelope (i.e. hepatitis $\mathrm{B}$ envelope antigen, $\mathrm{HBeAg}$ ) onto which surface proteins (i.e. HBsAg) are embedded. This encases an inner nucleocapsid 'core', where the viral genome exists in a partially doublestranded, circular configuration, without the typical covalent bonds between the positive (+) and negative (-) strands. This is commonly termed incomplete 'relaxed circular' DNA.

\section{HBV replicative life cycle}

$\mathrm{HBV}$ attaches to and gains entry into host cells (i.e. hepatocytes) through interactions between $\mathrm{HBsAg}$ and cellular surface receptors - sodium/taurocholate co-transporting polypeptide. ${ }^{(4)}$ The outer envelope is shed as the nucleocapsid core gains entry into the cytoplasm, while the nucleocapside core is shed as the HBV DNA is transported into the host cell nucleus. Within the

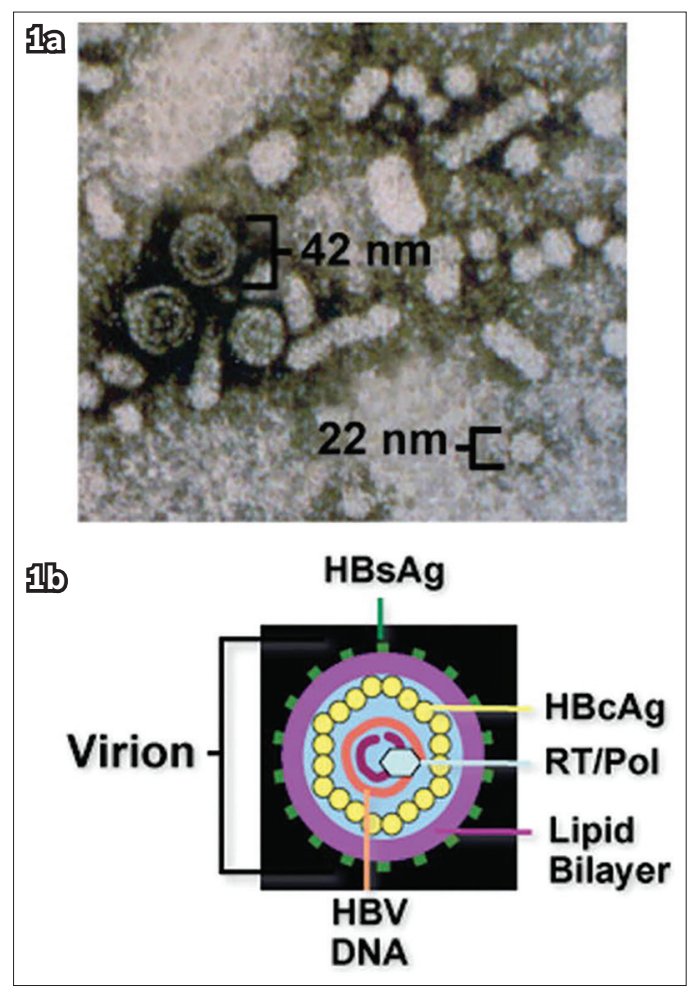

Fig. 1 (a) Electron micrograph shows the circulating forms of hepatitis B virion and subviral particles in blood, with a size range of 22-42 nm. (b) Schematic diagram shows the structure of the hepatitis B virus [reproduced with permission of Wiley and Sons from Liang $\mathrm{TJ}^{(26)}$ ]. HBcAg: hepatitis B core antigen; HBsAg: hepatitis B surface antigen; HBV: hepatitis $B$ virus; Pol: polymerase; RT: reverse transcriptase

${ }^{1}$ Department of Gastroenterology and Hepatology, Singapore General Hospital, ${ }^{2}$ Singapore Armed Forces Medical Corps, ${ }^{3}$ Duke-NUS Medical School, Singapore Correspondence: Dr Clarence Kwan Kah Wai, Department of Gastroenterology and Hepatology, Singapore General Hospital, Outram Road, Singapore 169608. clarence.kwan.k.w@singhealth.com.sg 


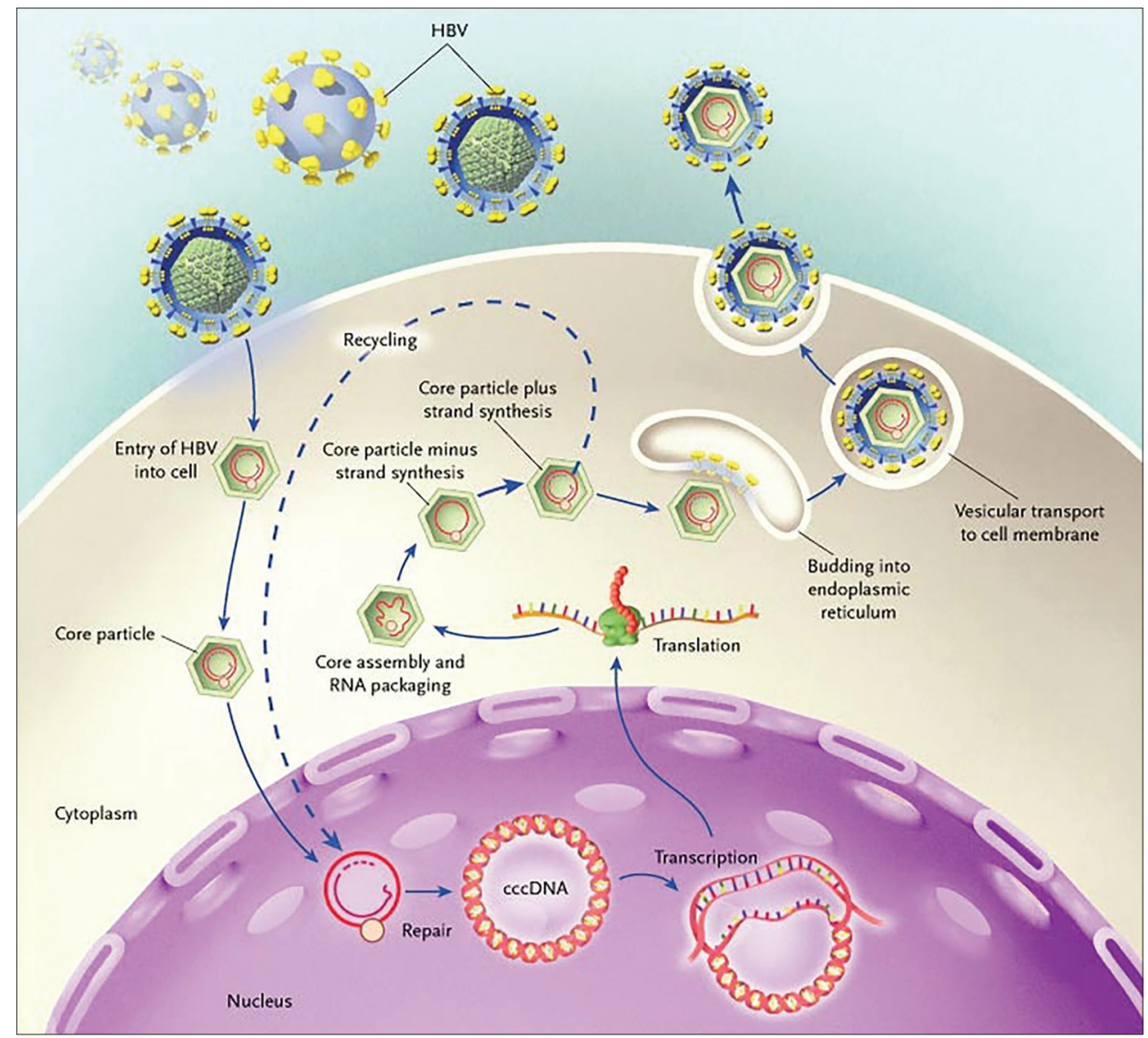

Fig. 2 Illustration shows the hepatitis B virus (HBV) replicative life cycle. Hepatitis B virions bind to surface receptors and are internalised. Viral core particles migrate to the hepatocyte nucleus, where their genomes are paired to form covalently closed circular DNA (cccDNA), which is the template for viral messenger RNA (mRNA) transcription. The viral mRNA that results is translated in the cytoplasm to produce the viral surface, core, polymerase and $X$ proteins. There, progeny viral capsids assemble, incorporating genomic viral RNA (RNA packaging). This RNA is reverse transcribed into viral DNA. The resulting cores can either bud into the endoplasmic reticulum to be enveloped and exported from the cell or recycle their genomes into the nucleus for conversion into cccDNA. The small sphere inside the core particle is the viral DNA polymerase [reproduced with permission of Massachusetts Medical Society from Ganem et $\left.\mathrm{al}^{(5)}\right]$.

nucleus, the HBV genome first undergoes repair and structural changes catalysed by host DNA polymerase. This involves the addition of complementary nucleotides to the incomplete DNA strand and the creation of covalent bonds between the positive and negative DNA strands, with the end product being a complete cccDNA. ${ }^{(5,6)}$

The cccDNA then serves as a functional template for the formation of translational messenger RNA (coding for viral proteins), as well as pre-genomic RNA (an anti-sense 'mirror' from which additional copies of relaxed circular DNA are formed). Viral subunits and relaxed circular DNA subsequently undergo packaging to form new HBV virions, which are then released into the bloodstream, infecting other hepatocytes and perpetuating the HBV infection(7) (see Fig. 2 for graphical illustration).

\section{HBV SEROLOGY}

$\mathrm{HBV}$ is able to escape host immune detection through a series of mechanisms that cause chronic infection. ${ }^{(8)}$ This will not be covered here, as it is beyond the scope of the article. Notwithstanding this, antigenic stimuli exerted by circulating virions will eventually trigger a response from the host's adaptive immune system, resulting in the production of antibodies against the viral proteins.
The role of antibodies to HBV envelope protein (anti-HBe) is not clear. The appearance of this antibody may not be initially detected by serological assays because it exists as antigen-antibody complexes with $\mathrm{HBeAg}$. The latter is produced in extremely high quantities and 'overwhelms' the anti-HBe. ${ }^{(5)}$ The appearance of anti-HBe and disappearance of HBeAg are hence related to a decreased production of viral proteins and are indirect markers of the transition from an active HBV replicative state to a quiescent, low replicative state. This is confirmed by a low or undetectable HBV DNA level. ${ }^{5}$

Similar to anti-HBe, antibodies to the nucleocapsid core (i.e. anti-HBc) are 'non-neutralising' antibodies that do not confer immune protection. Anti-HBc can be detected early in the course of HBV infection and typically persists lifelong. Given this characteristic, it is commonly regarded as a 'serological

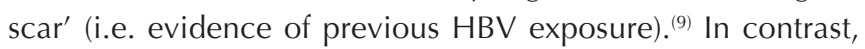
antibodies against HBsAg (anti-HBs) neutralise virions by inhibiting their ability to gain entry into host cells, thus preventing HBV infection. Individuals who achieve anti-HBs seroconversion following $\mathrm{HBV}$ infection will typically have no detectable HBV DNA in the serum. The expected sequence of appearance of serologic markers in acute and chronic HBV infection is depicted in Fig. 3. 

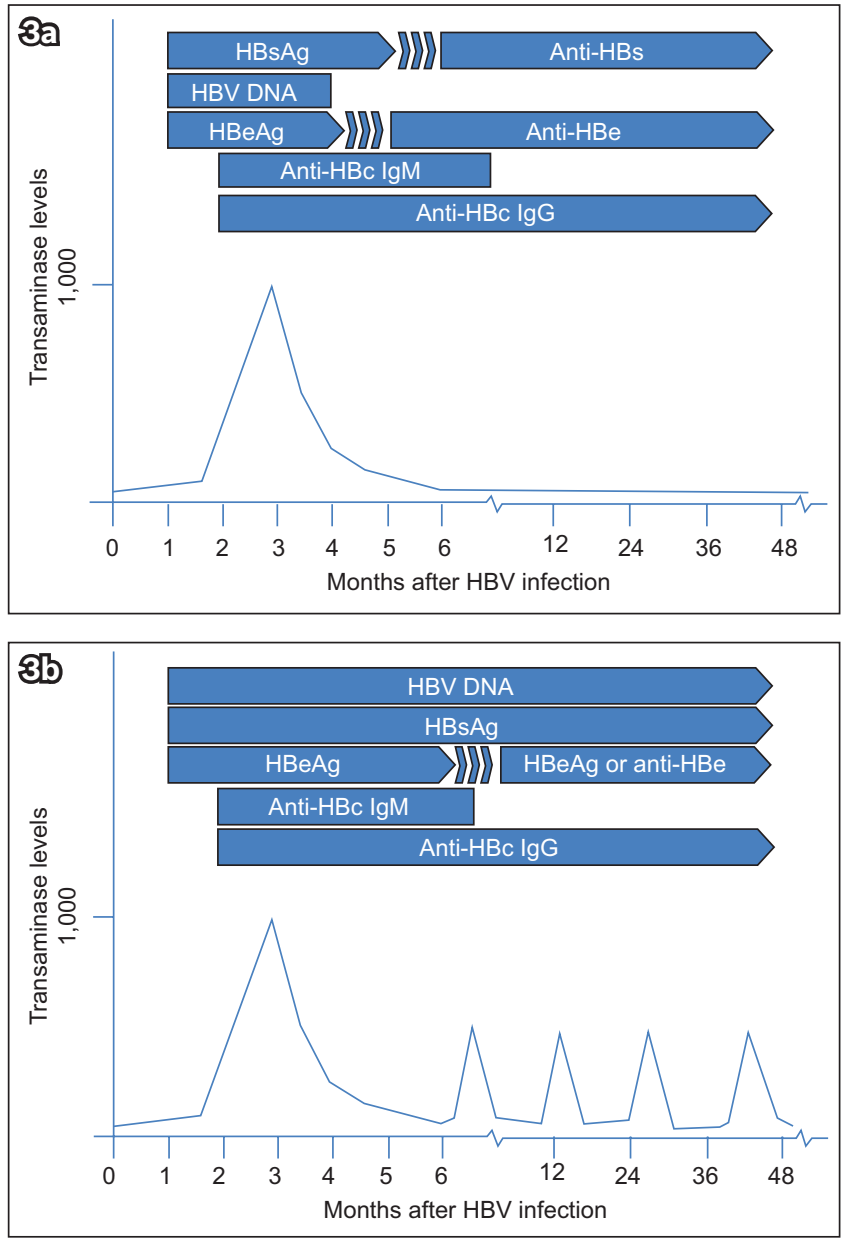

Fig. 3 Diagrams show the sequence of appearance of serologic markers (a) during acute hepatitis B virus infection and (b) transition of acute to chronic infection. Anti-HBc: antibodies to hepatitis B core protein; anti-HBe: antibodies to hepatitis B envelope antigen; anti-HBs: antibodies to hepatitis B surface antigen; HBeAg: hepatitis B envelope antigen; HBsAg: hepatitis B surface antigen; HBV: hepatitis B virus; IgG: immunoglobulin G; IgM: immunoglobulin M

\section{OCCULT HBV INFECTION}

HBV nuclear cccDNA molecules have a chromatin-like structure and can exist within the nucleus as a stable viral minichromosome. ${ }^{(10)}$ The transcriptional, and thus replicative, activity of HBV cccDNA is dependent on poorly understood interactions between 'activating factors' (such as the HBV X protein) and epigenetic control by the host cell. ${ }^{(11,12)}$ This is part of the virological key that explains the continued persistence of HBV.

Therefore, those who have had previous HBV infection (i.e. anti-HBc positive) with a reservoir of HBV cccDNA within the hepatocytes run a perpetual risk of viral reactivation despite anti-HBs seroconversion and HBV DNA clearance from the serum. Such persons are deemed to have OBI; i.e. detectable HBV DNA in the liver (ccCDNA in this case) of HBsAg-negative individuals regardless of serum HBV DNA and anti-HBs status. ${ }^{(13)}$

Patients with $\mathrm{OBI}$ should not be confused with those harbouring HBV 'S escape mutants', as the latter typically have modified HBsAg that is not recognised by commercially available detection assays. ${ }^{(14)}$ In addition, they would have positive anti-HBC and falsely negative HBsAg results. However, the most obvious distinguishing feature is the typically high serum HBV DNA level, which is comparable to that seen in patients with chronic HBV infection.

\section{Natural history of patients with OBI}

Little is known about the natural history of patients with OBI. Chemin et al retrospectively reviewed the serum HBV DNA level of 26 patients with cryptogenic hepatitis who were negative for $\mathrm{HBsAg}$ and positive for anti-HBc antibodies. Over the period of follow-up, all had transient episodes of HBV DNA positivity, albeit at low titres $<1 \times 10^{4}$ copies $/ \mathrm{mL}$. There was no relationship between alanine transaminase levels and HBV DNA detectability. ${ }^{(15)}$

\section{Prevalence of $\mathrm{OBI}$ among anti-HBc positive patients}

Some authors have attempted to confirm the presence of $\mathrm{OBI}$ by measuring the HBV DNA level in patients with positive anti-HBc, and found a frequency of HBV DNA seropositivity of $0.56 \%-8.00 \%{ }^{(16)}$ In a similar attempt, Tandoi et al analysed the liver tissue of patients who were negative for HBsAg and positive for anti-HBc, and found that up to $60 \%$ of patients had detectable cccDNA. In this cohort, serum HBV DNA was positive in only $17 \%$ of patients with detectable cccDNA. ${ }^{(17)}$ At present, the turnover kinetics of HBV cccDNA remains unclear. However, viral reactivation can occur even among those with resolved $\mathrm{HBV}$ infection in the previous decades, reflecting the extremely slow rate of clearance of HBV cccDNA. ${ }^{(18,19)}$

\section{ANTI-HBC DONORS: A POTENTIAL SOURCE OF DE NOVO HBV INFECTION?}

In the context of immunosuppression, intermittent increases in HBV cccDNA transcriptional activity and release of hepatitis B virions into the bloodstream may not be effectively contained by a weakened immune system. In view of this, the transplantation of liver grafts from donors with $\mathrm{OBI}$ poses a potential risk of DNHI to the recipients, due to the near-universal use of immunosuppressive medications to prevent graft rejection. Intuitively, the ideal approach would be to further risk stratify the cohort with positive anti-HBc antibodies, so as to identify and exclude those with $\mathrm{OBI}$ from organ donation, thus eliminating the risk of cross infection. Unfortunately, to assess liver tissue for cccDNA mandates a liver biopsy, and there is still no standardised or easily applicable method for quantifying cccDNA in hepatocytes. The laboratory equipment and expertise required is neither cheap nor easily available.

A systematic review conducted by Cholongitas et al on recipients receiving liver grafts with positive anti-HBc concluded that the risk of $\mathrm{DNHI}$ is highest among recipients who are naïve to HBV infection (47.8\%). ${ }^{(20)}$ Their findings on recipients who did not receive post-transplant prophylaxis are summarised in Fig. 4. This is supported by Skagen et al, who showed a rate of DNHI of 58\% in HBV-naïve liver transplant recipients. ${ }^{(21)}$ The fact that the risk of $\mathrm{DNHI}$ in HBV-naïve recipients so closely mirrors the prevalence of $\mathrm{OBI}$ among patients with positive anti-HBc antibodies suggests that $\mathrm{DNHI}$ may be an inevitable event in such circumstances. In 


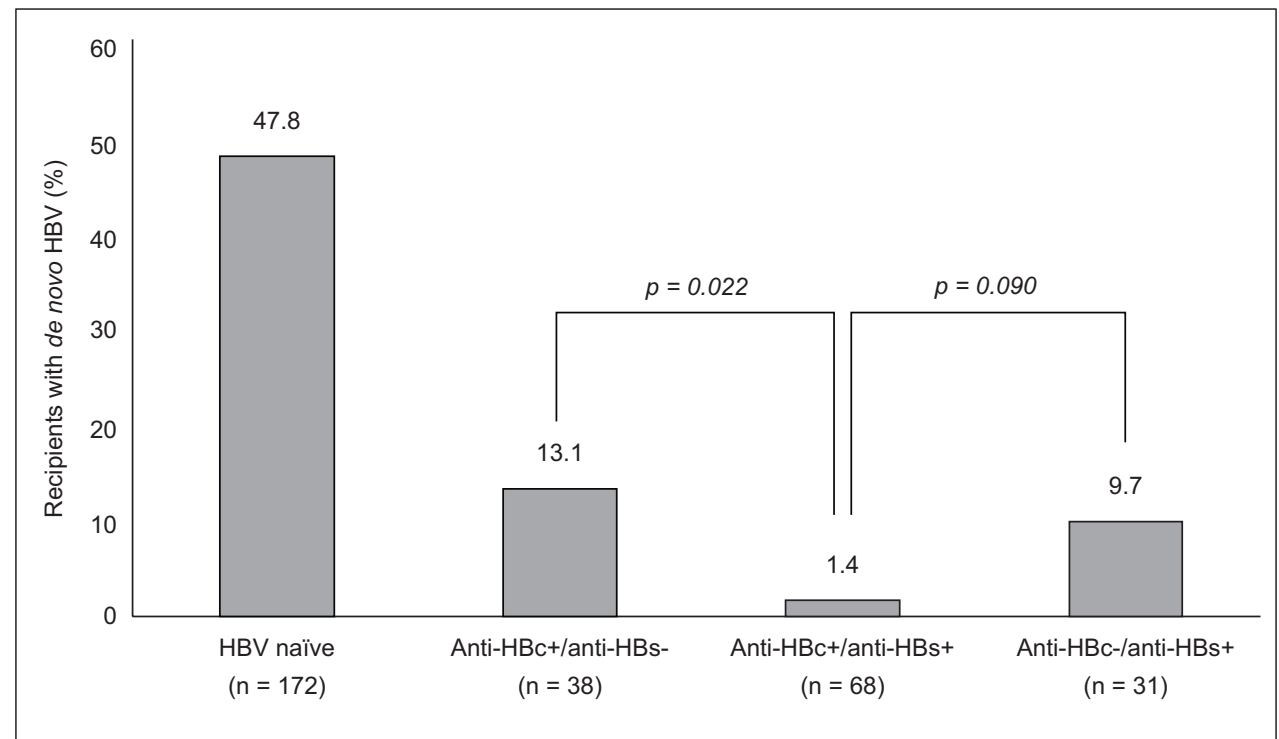

Fig. 4 Charts shows the risk of de novo hepatitis B virus (HBV) infection in patients of different HBV serological status who receive anti-HBc+ liver grafts, based on data presented by Cholongitas et al. ${ }^{\left({ }^{20}\right)} \mathrm{p}<0.001$ for all comparisons against HBV naïve patients. Anti-HBc-: antibodies to hepatitis $B$ core protein negative; anti-HBc+: antibodies to hepatitis B core protein positive; anti-HBs-: antibodies to hepatitis B surface antigen (HBsAg) negative; anti-HBs+: positive antibodies to HBsAg positive

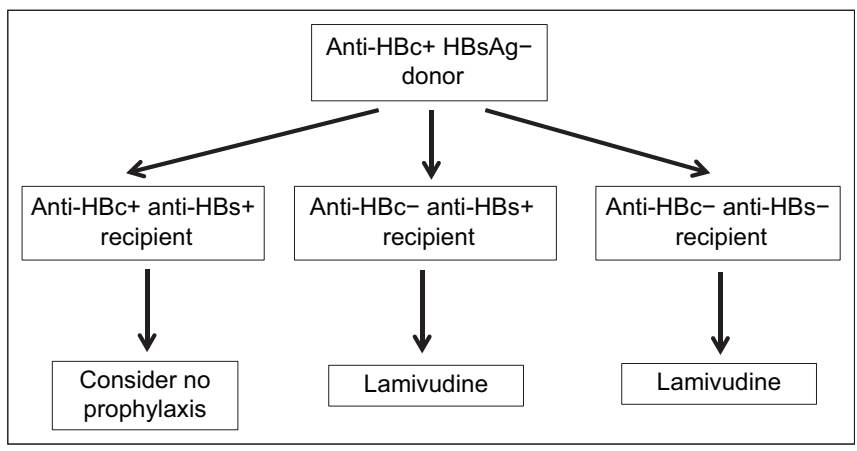

Fig. 5 Flowchart shows the flow of decision-making for choice of immunophylaxis [reproduced with permission of Wiley and Sons from Huprikar et $\left.\mathrm{al}^{(23)}\right]$.

contrast, recipients who had acquired natural immunity were at the lowest risk of DNHI (1.4\%-4.0\%). Patients who were anti-HBc positive/anti-HBs negative or anti-HBc negative/ anti-HBs positive demonstrated intermediate risks of $\mathrm{DNH}$ of $9.7 \%-18.0 \% .^{(20,21)}$

\section{PRACTICAL MANAGEMENT OF ANTI-HBC DONORS AND DNHI RISK}

Patients with natural immunity do not require immunoprophylaxis due to the low rates of DNHI and can be followed up with serial measurement of HBV DNA levels. Immunoprophylaxis should be considered in other groups of patients due to their significant risks of DNHI.

Three main strategies have been used to reduce the risk of DNHI: (a) hepatitis B immunoglobulin (HBIG); (b) nucleoside analogues (mainly lamivudine); and (c) a combination of the two. HBIG monotherapy has only been shown to have marginal benefits in preventing $\mathrm{DNHI}$ and thus should not be used as such. ${ }^{(20,21)}$ The risk of DNHI was reduced from $9.7 \%-18.0 \%$ to $0 \%-4 \%$ with the use of lamivudine with or without $\mathrm{HBIG}$ immunoprophylaxis. ${ }^{(20,21)}$ In a separate systematic review, Saab et al demonstrated that combination therapy of HBIG and lamivudine $(4 / 110,3.6 \%)$ did not result in better risk reduction of DNHI, as compared to lamivudine monotherapy (2/73, 2.7\%).(22) The American and Canadian Societies of Transplantation have jointly published a set of consensus guidelines for the posttransplant management of patients receiving solid organs from anti-HBc positive donors grafts. ${ }^{(23)}$ A summary of the guidelines is shown in Fig. 5.

Lamivudine is the most extensively evaluated nucleoside analogue and is recommended as the most cost-effective option for reducing the risk of DNHI. Antiviral resistance, typically a concern with long-term lamivudine therapy, is less commonly encountered in the context of post-transplant prophylaxis; this is likely related to the fact that cccDNA exists in the low replicative state and HBV DNA titres (if at all detected in the serum) tend to be low. ${ }^{(24)}$ Despite this, newer nucleoside analogues with high genetic barrier to resistance are increasingly being used today. Although large-scale clinical data and information on the longterm cost-effectiveness of entecavir and tenofovir are still lacking, their use in this context has been associated with favourable results in preventing $\mathrm{DNHI} .{ }^{(25)}$

\section{CONCLUSION}

In conclusion, there is a high prevalence of $\mathrm{OBI}$ among individuals with anti-HBc antibodies. Nevertheless, liver grafts from these patients can be safely utilised if appropriate recipient matching and adequate post-transplant prophylaxis are performed. The ideal prophylactic regime for the prevention of DNHI is not clearly defined due to the lack of robust data and varied practice across different institutions. Although there seems to be a paradigm shift from long-term immunoprophylaxis to nucleoside analogue monotherapy without immunoprophylaxis, this requires further validation. 


\section{REFERENCES}

1. Pang M. Number of patients waiting for liver transplant triples. The Straits Times 2012 Jun 25.

2. Ang LW, Cutter J, James L, Goh KT. Seroepidemiology of hepatitis B virus infection among adults in Singapore: a 12-year review. Vaccine 2013; 32:103-10.

3. Dane DS, Cameron $\mathrm{CH}$, Briggs M. Virus-like particles in serum of patients with Australia-antigen-associated hepatitis. Lancet 1970; 1:695-8.

4. Nguyen DH, Ludgate L, Hu J. Hepatitis B virus-cell interactions and pathogenesis. J Cell Physiol 2008; 216:289-94.

5. Ganem D, Prince AM. Hepatitis B virus infection-natural history and clinical consequences. N Engl J Med 2004; 350:1118-29.

6. Tuttleman JS, Pourcel C, Summers J. Formation of the pool of covalently closed circular viral DNA in hepadnavirus-infected cells. Cell 1986; 47:451-60.

7. Beck J, Nassal M. Hepatitis B virus replication. World J Gastroenterol 2007; 13:48-64.

8. Ferrari C. HBV and the immune response. Liver Int 2015; 35 Suppl 1:121-8.

9. Lok AS, McMahon BJ. Chronic hepatitis B: update 2009. Hepatology 2009; 50:661-2.

10. Levrero M, Pollicino T, Petersen J, et al. Control of cccDNA function in hepatitis B virus infection. J Hepatol 2009; 51:581-92.

11. Murr R. Interplay between different epigenetic modifications and mechanisms. Adv Genet 2010; 70:101-41.

12. Zhang $\mathrm{T}$, Xie N, He W, et al. An integrated proteomics and bioinformatics analyses of hepatitis $B$ virus $X$ interacting proteins and identification of a novel interactor apoA-I. J Proteomics 2013; 84:92-105.

13. Raimondo G, Allain JP, Brunetto MR, et al. Statements from the Taormina expert meeting on occult hepatitis B virus infection. J Hepatol 2008; 49:652-7.

14. Purdy MA. Hepatitis B virus S gene escape mutants. Asian J Transfus Sci 2007; $1: 62-70$.

15. Chemin I, Guillaud O, Queyron PC, Trépo C. Close monitoring of serum HBV
DNA levels and liver enzymes levels is most useful in the management of patients with occult HBV infection. J Hepatol 2009; 51:824-5.

16. Ye X, Li T, Xu X, et al. Characterisation and follow-up study of occult hepatitis B virus infection in anti-HBc-positive qualified blood donors in southern China. Blood Transfus 2016; 15:6-12.

17. Tandoi F, Caviglia GP, Pittaluga F, et al. Prediction of occult hepatitis B virus infection in liver transplant donors through hepatitis B virus blood markers. Dig Liver Dis 2014; 46:1020-4.

18. Hoofnagle JH. Reactivation of hepatitis B. Hepatology 2009; 49(5 Suppl):S156-65.

19. Kumar R, Pérez-Del-Pulgar S, Testoni B, Lebossé F, Zoulim F. Clinical relevance of the study of hepatitis B virus covalently closed circular DNA. Liver Int 2016; 36 Suppl 1:72-7.

20. Cholongitas E, Papatheodoridis GV, Burroughs AK. Liver grafts from antihepatitis B core positive donors: a systematic review. J Hepatol 2010; 52:272-9.

21. Skagen $\mathrm{CL}$, Jou JH, Said A. Risk of de novo hepatitis in liver recipients from hepatitis-B core antibody-positive grafts - a systematic analysis. Clin Transplant 2011; 25:E243-9.

22. Saab S, Waterman B, Chi AC, Tong MJ. Comparison of different immunoprophylaxis regimens after liver transplantation with hepatitis B core antibody-positive donors: a systematic review. Liver Transpl 2010; 16:300-7.

23. Huprikar S, Danziger-Isakov L, Ahn J, et al. Solid organ transplantation from hepatitis B virus-positive donors: consensus guidelines for recipient management. Am J Transplant 2015; 15:1162-72.

24. Leong J, Coty P, Fiel MI, et al. Lamivudine resistance leading to de novo hepatitis B infection in recipients of hepatitis B core antibody positive liver allografts. Hepatol Res 2014; 44:1248-52.

25. Chang MS, Olsen SK, Pichardo EM, et al. Prevention of de novo hepatitis B in recipients of core antibody-positive livers with lamivudine and other nucleos(t) ides: a 12-year experience. Transplantation 2013; 95:960-5.

26. Liang TJ. Hepatitis B: the virus and disease. Hepatology 2009; 49(5 Suppl):S13-21. 